\title{
Bem-estar em equinos de policiamento em Curitiba/PR: indicadores clínicos, etológicos e ritmo circadiano do cortisol
}

\author{
Welfare in police horses from Curitiba/PR, Brazil: \\ clinical and behavior indicators and circadian cortisol rhythm.
}

\author{
Lilian D'Almeida Gontijo ${ }^{\mathrm{I}}$ Fabiane Cassou ${ }^{\mathrm{I}}$ Pedro Vicente Michelotto Junior ${ }^{\mathrm{II}}$ \\ Geraldo Eleno Silveira Alves ${ }^{\mathrm{I}}$ Beatriz Bringel ${ }^{\mathrm{III}}$ Rodrigo Martins Ribeiro ${ }^{\mathrm{I}}$ \\ Luiz Alberto do Lago $^{\mathrm{I}}$ Rafael Resende Faleiros ${ }^{\mathrm{I}}$
}

\section{RESUMO}

A avaliação científica do bem-estar tem sido importante para detectar animais não adaptados às condições de criação que lhes são impostas, situação que afeta o potencial produtivo e os coloca em maior risco de enfermidades. Com esse objetivo, foram avaliados 30 equinos da Cavalaria da Polícia Militar do Paraná, considerando-se indicadores clínicos, etológicos e hormonais. Na avaliação clínica, além da determinação do escore corporal e exame físico, verificou-se o histórico de ocorrência de cólica nos últimos doze meses. Comportamentos anormais foram pesquisados por observação etológica e a taxa do ritmo circadiano do cortisol (RCC) foi determinada por meio de duas colheitas de sangue, às 08 e às 17 horas. Apesar da significativa ocorrência de comportamentos anormais (26,7\%), os achados clínicos foram normais e houve ausência de relato de cólica nos últimos 12 meses precedentes ao estudo. As médias dos resultados das análises de cortisol estiveram dentro da normalidade, nos dados absolutos e na variação do RCC, entretanto, $42 \%$ dos animais analisados apresentaram RCC alterado. Apesar da expressiva incidência de comportamentos anormais e de animais com alteração no ritmo circadiano de cortisol, sinais indicadores de estresse crônico, considerou-se que os equinos deste estudo apresentaram boas condições de bem-estar em relação àquelas relatadas por estudos nacionais prévios.

Palavras-chave: estabulação, estresse, cavalo, comportamento, estereotipias.

\section{ABSTRACT}

The scientific assessment of welfare has been important to detect animals non adapted to their management conditions, which can interfere with their productive potential and put them at increased risk of illness. With this objective, 30 horses from the Military Police of Paraná were evaluated, considering clinical, ethological and hormonal indicators of equine welfare.
In the clinical evaluation, horses were assessed by physical examination and their body score conditions and the history of colic incidence in the last twelve months were determined. Abnormal behavior incidence were studied by ethological observation and the individual rate of circadian rhythm of cortisol (RCC) was calculated based on cortisol concentration changes in two blood samples taken at 08:00 am and 5:00pm. Despite significant incidence of abnormal behaviors (26.7\%), clinical findings were normal and there were no reports of colic episodes in the last 12 months preceding the study. The mean blood cortisol concentrations and the mean RCC rate were within the normal range. However, $42 \%$ of the studied horses had abnormal RCC rates. Despite the significant incidences of abnormal behavior and abnormal RCC rates (indicators of chronic stress), these horses were considered in good welfare when compared to ones previously reported in other national studies.

Key words: stress, housing, equine, behavior, stereotypies.

\section{INTRODUÇÃO}

A preocupação com o bem-estar animal está cada vez mais em pauta no Brasil e no mundo. Vários setores da sociedade têm se organizado no sentido de reivindicar melhores condições para a criação de animais. Contudo, na maioria das vezes, observam-se argumentos de grande cunho emocional e totalmente desprovidos de evidências científicas. Assim, pesquisadores, médicos veterinários e zootecnistas têm se empenhado em usar o método científico para detectar situações que interferem na qualidade de vida dos animais e propor

'Departamento de Clínica e Cirurgia Veterinárias (DCCV), Universidade Federal de Minas Gerais (UFMG), Av. Antônio Carlos, 6627,

CP 567, Campus Pampulha, 30161-970, Belo Horizonte, MG, Brasil. E-mail: faleirosufmg@gmail.com. *Autor para correspondência.

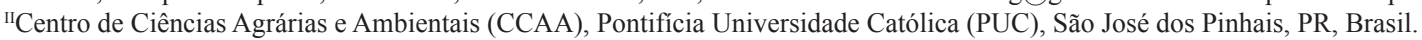

"IIBET Laboratórios, Rio de Janeiro, RJ, Brasil. 
medidas que favoreçam seu conforto e bem-estar, visando à convivência harmônica homem-animal. Sinais de bem-estar precário são evidenciados por mensurações fisiológicas, como aumento de frequência cardíaca ou alteração na atividade adrenal. Adoção de comportamentos anormais, presença de doença, ferimento, dificuldade de movimento, reduzida possibilidade de se exercitar e anormalidades de crescimento também são sinais de bem estar pobre (ANDERSON et al., 1999; BROOM \& MOLENTO, 2004).

Estudos científicos conduzidos por nosso grupo revelaram que equinos que trabalham com patrulhamento em ambiente urbano podem estar sujeitos a elevada incidência de anomalias de comportamento e cólicas (LEAL, 2007; LEAL et al., 2011). Nos equinos de patrulhamento da região metropolitana de Belo Horizonte, detectou-se associação entre esses fatores e alterações no ritmo circadiano de cortisol, evidenciando que o estresse crônico seria a principal causa dessas alterações (LEAL, 2007; LEAL et al., 2011).

O objetivo do estudo foi avaliar o bemestar de equinos submetidos ao patrulhamento urbano sob regime de estabulação na cidade de Curitiba/ $\mathrm{PR}$, por meio de indicadores clínicos, etológicos e endocrinológicos.

\section{MATERIAL E MÉTODOS}

Foram utilizados 30 equinos, machos e fêmeas, com idade média $( \pm \mathrm{DP})$ de $15 \pm 3,7$ anos, estabulados na cidade de Curitiba, na Cavalaria da Polícia Militar do Paraná (Regimento de Polícia Montada Coronel Dulcídio). Dentro da avaliação clínica, classificou-se a condição corporal (adiposidade) utilizando-se uma escala de 1 a 9, sendo 1 caquético e 9 o animal extremamente obeso, conforme previamente descrito (CARROLL \& HUNTINGTON, 1988). Logo após, realizou-se uma avaliação do estado geral do animal, baseando-se na qualidade do pelame, crina e casco, presença de alterações de saúde evidentes, a fim de verificar se o animal estava bem cuidado ou não. $\mathrm{O}$ estado geral foi classificado em 5 graus, sendo o grau 1 péssimo, 2 ruim, 3 regular, 4 bom ou grau 5, ótimo. Posteriormente, foi feita avaliação quanto à presença de feridas escoriações com classificação de 0 a 4 , sendo 0 ausente e o grau 4 com maior intensidade de lesões.

No exame físico, avaliou-se a frequência cardíaca, com o auxílio de estetoscópio, e a frequência respiratória, realizada por contagem visual dos movimentos torácicos e auscultação com estetoscópio. Também como parte da avaliação de saúde dos equinos, verificou-se a ocorrência de cólicas nos últimos 12 meses, examinando-se as fichas individuais de clínica veterinária da Polícia Militar.

Para se verificar a ocorrência de estereotipias, a observação de todos os animais foi feita na primeira semana de outubro de 2009, sempre pela mesma pesquisadora, e de forma individual. $\mathrm{O}$ período de observação de cada animal foi de 6 horas de um mesmo dia, sendo quatro horas durante o dia e duas horas durante a noite (LEAL, 2007, modificado). A avaliação foi realizada com os animais em seu próprio ambiente, mantendo uma distância entre a pesquisadora e o animal para que não se interessasse pela presença humana. Foram considerados os tipos de comportamentos classificados como normais (distraído, alerta em estação, deitado, focinho rente ao chão e relinchar) ou anormais (agressividade, cavar, caminhada estereotípica pela baia, movimentos repetidos de cabeça, dança de lobo ou síndrome do urso (weaving), aerofagia, morder madeira, coprofagia, lambedura de cocho).

Adicionalmente, para análise do comportamento, foi acessado o temperamento dos equinos por meio de entrevista com o tratador, sendo preenchido um questionário. Nesse questionário, o temperamento foi classificado nos graus 1 a 3, sendo o grau 1 de menor intensidade e o grau 3 de maior intensidade. Os comportamentos sugeridos foram brincalhão, curioso, nervoso e assustado (LEAL, 2007). Com objetivo de verificar o ritmo circadiano do cortisol, colheram-se duas amostras de sangue de cada um dos animais, às $8 \mathrm{~h}$ e às $17 \mathrm{~h}$. Após desinfecção do local com álcool iodado, foi feita a punção da jugular, utilizando-se agulhas descartáveis e frascos dotados de sistema a vácuo. Cada colheita foi realizada com o animal em seu próprio ambiente, com a ajuda do tratador ou pessoa de contato próximo do animal, e em tempo inferior a 30 segundos, minimizando a ocorrência de estresse agudo a ele. As amostras de sangue foram então centrifugadas e o soro separado e congelado à temperatura de $-20^{\circ} \mathrm{C}$. Para análise do cortisol sorológico, foi utilizada a técnica de radioimunoensaio (RIE), de acordo com o protocolo do laboratório conveniado (Bet Laboratórios).

Foi utilizado o método proposto por DOUGLAS (2000), (valor maior - menor) / valor maior, para definir a taxa do ritmo circadiano do cortisol (RCC) de cada animal. Se o RCC fosse inferior a 0,30, considerou-se que o RCC estivesse alterado. Os dados foram tabulados, obtendo-se as médias e os desvios padrão para os dados paramétricos e as frequências absolutas e percentuais para as ocorrências dos diversos tipos de comportamentos, 
temperamentos, cólicas e RCC alterados. Os escores para temperamento foram comparados entre si pelo teste de Kruskal-Wallis e as prevalências de escores com intensidade máxima (3) pelo texto de Chi-quadrado. Possíveis correlações entre escores de temperamento ou presença de comportamentos anormais com concentrações séricas de cortisol, taxa de RCC e frequências cardíaca e respiratória foram acessadas pelo teste de Spearman. Para todos os testes, considerou-se $\mathrm{P} \leq 0,05$.

\section{RESULTADOS E DISCUSSÃO}

Durante a observação, os animais apresentaram médias de frequências cardíaca e respiratória dentro dos parâmetros normais para a espécie (Tabela 1). A condição geral dos equinos foi classificada como adequada, com baixo índice de escoriações e sem incidência de animais magros (Tabela 1).

As variáveis fisiológicas estiveram dentro dos valores esperados para a espécie e se assemelharam aos valores observados em equinos da Polícia Militar de Belo Horizonte (LEAL, 2007). $\mathrm{O}$ fato de que os equinos tiveram escore médio de 3,4 para estado geral e de que $80 \%$ dos animais foram classificados com lesões ausentes ou mínimas, demonstra que os animais estavam recebendo um bom tratamento nesta unidade militar.

A maioria dos animais apresentou escore corporal adequado (entre 6 e 7), contudo cinco animais foram classificados como obesos (escores 8 e 9). A obesidade não é uma situação desejada em equinos, pois aumenta dos riscos de doenças graves como hiperlipidemia e laminite (JOHNSON et al., 2009). O sistema aqui utilizado para classificar a condição corporal de 1 a 9 já se mostrou útil em predizer hiperinsulinemia e risco de laminite em pôneis (CARTER et al., 2009), podendo assim ser utilizado para monitorar equinos de forma a evitar que eles se tornem obesos, situação que traz prejuízo à saúde e ao bem-estar.

De acordo com a opinião dos tratadores, observou-se prevalência de máxima intensidade (3) de $40 \%$ de assustados, $36 \%$ de curiosos, $30 \%$ de nervosos e 16\% de brincalhões. Entretanto, tais incidências, nem tampouco os escores médios para cada temperamento (Tabela 2), não diferiram entre si $(\mathrm{P}>0,05)$. Considerando-se todos os tipos de comportamentos anormais, verificou-se que $26,7 \%$ dos equinos apresentaram algum tipo de distúrbio. Agressividade foi o mais relatado $(13,3 \%)$, seguido de coprofagia $(6,7 \%)$, movimentos repetidos de cabeça $(6,7 \%)$ e movimentos aleatórios (3,3\%), conforme descrito na tabela 3 .

A incidência total de comportamentos anormais de $26,7 \%$ esteve próxima dos limites relatados na literatura internacional para equinos estabulados de 7 a 30\% na Austrália (JOHNSON et al., 1998) e de 5 a $20 \%$ na Europa (MILLS \& NANKERVIS, 2005). Entretanto, foi inferior às incidências relatadas para animais estabulados em unidades militares brasileiras: 28,9 \% em Brasília, DF (VIEIRA, 2006), e 43\% em Belo Horizonte, MG (LEAL, 2007), como também ao valor de 32,5\%, descrito por MCGREEVY et al. (1995) em equinos estabulados submetidos à atividade de adestramento. A principal alteração de comportamento observada neste estudo foi agressividade, relatada em quatro dos trinta animais estabulados.

Outro aspecto positivo da avaliação comportamental no presente trabalho foi a ausência de aerofagia, que havia sido relatada em outras unidades militares em Minas Gerais, (3,6\%, LEAL, 2007) e Brasília 8,5\% (VIEIRA, 2006). Essa estereotipia geralmente é correlacionada à restrição de comportamentos de origem oral, como, por exemplo, ausência de pastejo em equinos estabulados (MCGREEVY, 2004). A ausência de aerofagia nos equinos militares do Paraná pode ser explicada pela qualidade e variedade do volumoso oferecido.

Um dado muito interessante encontrado nos equinos da Polícia Militar de Curitiba foi o índice nulo de cólicas nos doze meses antecedentes ao exame dos animais. Estudos realizados em animais estabulados em unidades militares no Brasil têm revelado números alarmantes. No Estado do Rio de Janeiro, relataram-se incidências de 12 a 95 episódios de cólica/100 animais/ano em três diferentes unidades do exército (LARANJEIRA et al., 2009). Já em unidades de Polícia Militar, a incidência anual de

Tabela 1 - Médias e desvios padrão dos graus observados para estado geral, condição corporal e presença de escoriações e dos valores das frequências cardíaca (FC) e respiratória (FR) em equinos militares em Curitiba, PR.

\begin{tabular}{lccccc}
\hline & Estado geral (1-5) & Condição Corporal (1-9) & Escoriações (0-4) & FC (bat min $\left.{ }^{-1}\right)$ & FR (mov min $\left.{ }^{-1}\right)$ \\
\hline Média & 3,40 & 6,53 & 0,60 & 41,19 & 16,67 \\
DP & 0,67 & 1,07 & 0,81 & 11,49 & 4,80 \\
\hline
\end{tabular}


Tabela 2 - Médias e desvios padrão dos graus de temperamento observados em equinos da Polícia Militar do Paraná estabulados na região urbana de Curitiba, em junho de 2009.

\begin{tabular}{lcccc}
\hline & Brincalhão (1-3) & Curioso (1-3) & Nervoso (1-3) & Assustado (1-3) \\
\hline Média & 1,57 & 2,03 & 1,80 & 1,93 \\
DP & 0,77 & 0,89 & 0,89 & 0,94 \\
\hline
\end{tabular}

cólicas na cavalaria em Brasília foi de 65 episódios de cólica 100 animais $^{-1}$ ano $^{-1}$ (VIEIRA, 2006) e, em Belo Horizonte, 92 episódios de cólica 100 animais $^{-1}$ ano $^{-1}$ (LEAL, 2007). Esses achados refletem a boa nutrição e o bom manejo dos animais do presente estudo.

Devido a armazenamento inadequado, oito amostras de sangue colhidas de manhã e oito colhidas de tarde não foram analisadas, o que possibilitou 12 leituras de RCC. Os valores médios para concentração de cortisol e de RCC se mantiveram dentro dos padrões de referência para a espécie (Tabela 4), contudo cinco dos 12 animais examinados apresentaram RCC acima de 0,30.

Cavalos confinados em unidade militar em Belo Horizonte apresentaram valores médios da taxa de RCC entre 0,18 a 0,26 (LEAL et al., 2011), ou seja, até duas vezes inferiores às médias apresentadas pelos cavalos do presente estudo $(0,36)$. Esses resultados, associados à ausência de cólica e menor incidência de distúrbios comportamentais indicam melhor nível de bem-estar dos animais dos grupos confinados em Curitiba. Apesar disso, 42\% dos animais examinados quanto ao RCC apresentaram índices abaixo do normal. A diminuição da taxa do RCC, ou seja, uma menor variação entre as concentrações matutinas e vespertinas de cortisol sérico é um indicativo de que o cavalo não está conseguindo enfrentar a situação estressante imposta a ele (ALEXANDER \& IRVINE, 1998). Por outro lado, alguns autores sugerem que a estereotipia seja uma forma que o animal encontra para lidar melhor com o estresse (COOPER \& NICOL, 1993; MCGREEVY \& NICOL, 1998). Há a hipótese de que, quando o equino apresenta comportamentos anormais e um RCC adequado, ele possa já ter se adaptado à situação e/ou ambiente estressor através da execução do movimento estereotípico (NICOL, 2000). Assim, animais que apresentam estereotipias, mesmo com concentrações normais de cortisol ou sem alteração na taxa de RCC, como verificado nesse estudo, podem já estar adaptados à situação, sendo a estereotipia uma parte do processo adaptativo.

Dentre os testes de Spearman, significância $(\mathrm{P}=0,03)$ foi obervada apenas para correlação negativa e baixa $(-0,41)$ entre o grau de temperamento brincalhão e a frequência respiratória. Apesar de pouco significado estatístico, esta correlação poderia ser um indicativo de que animais de temperamento brincalhão tenham menor atividade adrenérgica, refletindo em menor taxa respiratória. Outras correlações significativas não foram encontradas.

\section{CONCLUSÃO}

Equinos estabulados em ambiente urbano e em atividade de patrulhamento militar na cidade de Curitiba apresentaram incidência significativa de alterações comportamentais e de taxa de ritmo de circadiano de cortisol alterado, indicando situação de estresse crônico. Entretanto, esses equinos apresentaram condição corporal adequada, parâmetros

Tabela 3 - Incidência de comportamentos observados em equinos da Polícia Militar do Paraná estabulados na região urbana de Curitiba, em junho de 2009.

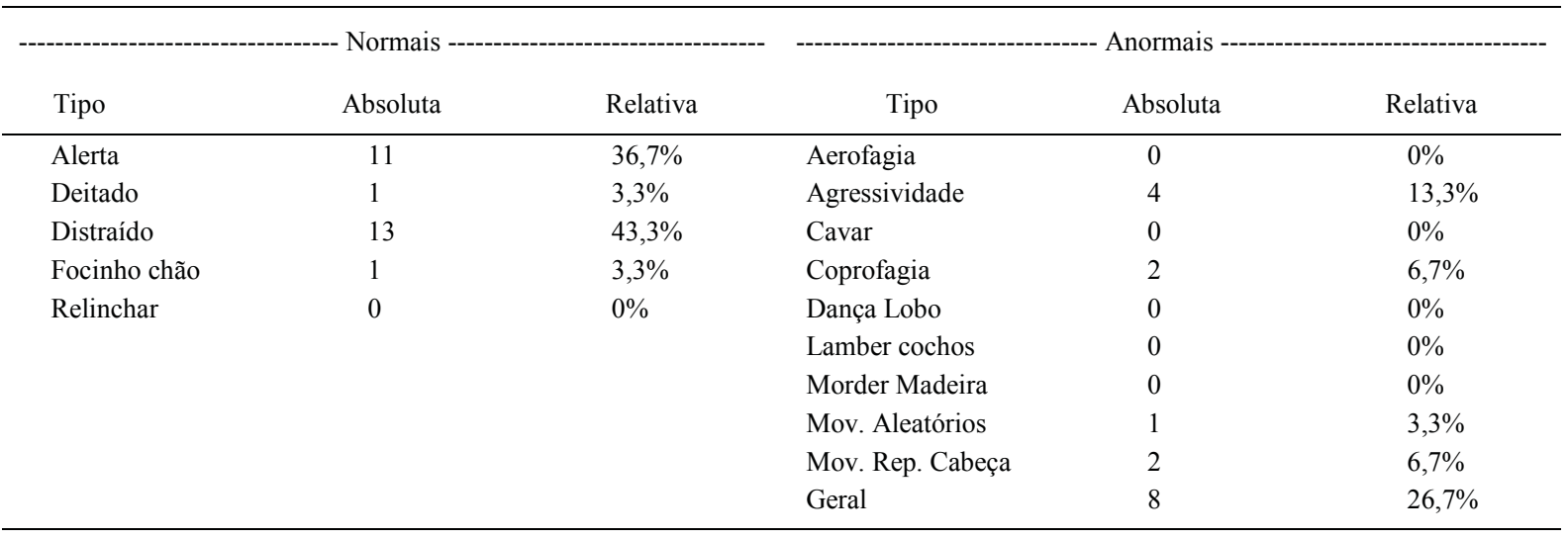


Tabela 4 - Médias e desvios padrão dos níveis de cortisol sérico matutino e vespertino e da taxa do ritmo circadiano de cortisol (RCC) em equinos da Polícia Militar do Paraná estabulados na região urbana de Curitiba, em junho de 2009.

\begin{tabular}{lccc}
\hline & & & \\
& Manhã $(\mathrm{n}=22)$ & Tarde $(\mathrm{n}=22)$ & $\begin{array}{c}\text { Taxa RCC } \\
(\mathrm{n}=12)\end{array}$ \\
\hline Média & 75,7 & 47,6 & 0,37 \\
DP & 23,9 & 29,0 & 0,28 \\
\hline
\end{tabular}

clínicos e concentrações médias de cortisol sanguíneo dentro dos valores de referência para a espécie e ausência de cólica no ano precedente ao exame. Dessa forma, considerou-se que, apesar dos indícios de estresse crônico, os equinos se apresentaram em condições de bem-estar acima das verificadas em estudos nacionais anteriores, considerando-se equinos estabulados em região urbana.

\section{AGRADECIMENTO}

Os autores agradecem ao Regimento de Polícia Montada Coronel Dulcídio, ao BET Laboratórios, pela análise das amostras, ao Conselho Nacional de Desenvolvimento Científico e Tecnológico (CNPq), Coordenação de Aperfeiçoamento de Pessoal de Nível Superior (CAPES), Simpósio Internacional do Cavalo Atleta (SIMCAV) e Fundação de Amparo à Pesquisa do estado de Minas Gerais (FAPEMIG) pelo financiamento do experimento.

\section{REFERÊNCIAS}

ALEXANDER, S.; IRVINE, C.H.G. Stress in the racing horse: coping vs not coping. Journal Equine Veterinary Science, v.9, p.77-81, 1998. Disponível em: <https://www.jstage.jst.go.jp/ article/jes/9/3/9_3_77/_article>. Acesso em: 02 nov. 2009. doi: $10.1294 /$ jes. $9.7 \overline{7}$.

ANDERSON, M.K. et al. Behavioral assessment of horses in therapeutic riding programs. AppliedAnimal Behaviour Science, v.63, p.11-24, 1999. Disponível em: <http://www.sciencedirect. com/science/article/pii/S0168159198002378>. Acesso em: 02 nov. 2009. doi: 10.1016/S0168-1591(98)00237-8.

BROOM, D.M.; MOLENTO, C.F.M. Bem-estar animal: conceitos e questões relacionadas - Revisão. Archives of Veterinary Science, v.9, n.2, p.1-11, 2004. Disponível em: $<$ http://www.unb.br/posgraduacao/docs/fav/MESTARANIMAL CONCEITOQUESTOESRELACIONADAS.pdf>. Acesso em: 02 nov. 2009. doi: ISSN: 1517-784X.

CARROLL, C.L.; HUNTINGTON, P.J. Body condition scoring and weight estimation of horses. Equine Veterinary Journal, v.20, p.41-45, 1988. Disponível em: <http://onlinelibrary.wiley. com/doi/10.1111/j.2042-3306.1988.tb01451.x/pdf>. Acesso em: 02 nov. 2009. doi: 10.1111/j.2042-3306.1988.tb01451.x.

CARTER, R.A. et al. Prediction of incipient pasture-associated laminitis from hyperinsulinaemia, hyperleptinaemia and generalised and localised obesity in a cohort of ponies. Equine Veterinary Journal, v.41, p.171-178, 2009. Disponível em:
<http://onlinelibrary.wiley.com/doi/10.2746/042516408X342975/ full>. Acesso em: 02 nov. 2009. doi: 10.2746/042516408X342975.

COOPER, J.J.; NICOL, C.J. The "coping" hypothesis of stereotypic behavior: a reply to Rushen. Animal Behaviour, v.45, p.616618, 1993. Disponível em: <http://www-sciencedirect-com.ez27. periodicos.capes.gov.br/science/article/pii/S0003347283710729>. Acesso em : 02 nov. 2009. doi: 003-3472/93/030616.

DOUGLAS, R.H. Circadian cortisol rhythmicity and equine Cushing's-like disease. Journal Equine Veterinary Science, v.19, p.684-753, 2000. Disponível em: <http://wwwsciencedirect-com.ez27.periodicos.capes.gov.br/science/article/ pii/S0737080699801117>. Acesso em: 02 nov. 2009. doi: 10.1016/S0737-0806(99)80111-7.

JOHNSON, K.G. et al. Behavioural changes in stabled horses given nontherapeutic levels of virginiamycin. Equine Veterinary Journal, v.30, n.2, p.139- 143, 1998.

JOHNSON, P.J. et al. Medical Implications of obesity in horses lessons for human obesity, Journal Diabetes Science Technology, v.3, p.163-174, 2009. Disponível em: <http://www.ncbi.nlm.nih. gov.ez27.periodicos.capes.gov.br/pmc/articles/PMC2769846/pdf/ dst-03-0163.pdf>. Acesso em: 02 nov. 2009. doi: PMC2769846.

LARANJEIRA, P.V.E. et al. Perfil e distribuição da síndrome cólica em eqüinos em três unidades militares do Estado do Rio de Janeiro, Brasil. Ciência Rural, v.39, p.1108-1115, 2009. Disponível em: $<$ http://www.scielo.br/pdf/cr/v39n4/a149cr945.pdf >. Acesso em: 02 nov. 2009. doi: 10.1590/S0103-84782009005000043.

LEAL, B.B. Avaliação do bem-estar dos equinos de cavalaria da Polícia Militar de Minas Gerais: Indicadores etológicos, endocrinológicos e incidência de cólica. 2007. 61f. Dissertação (Mestrado em Ciência Animal) - Escola de Veterinária da Universidade Federal de Minas Gerais, MG.

LEAL, B.B. et al. Cortisol circadian rhythm ratio: a simple method to detect stressed horses at higher risk of colic? Journal Equine Veterinary Science, v.31,p.188-190,2011. Disponível em: <http:// www.sciencedirect.com/science/article/pii/S0737080611000773>. Acesso em: 02 nov. 2009. doi: 10.1016/j.jevs.2011.02.005.

MCGREEVY, P.D. et al. Management factors associated with stereotypic and redirected behavior in thoroughbred horse. Equine Veterinary Journal, v.27, n.2, p.86-91, 1995.

MCGREEVY, P.; NICOL, C. Physiological and behavioral consequences associated with short-term prevention of crib-biting in horses. Physiology \& Behavior, v.65, n.1, p.15-23, 1998.

MCGREEVY, P.D. Equine behavior: a guide for veterinarians and equine scientists. London: Saunders. v. 169, f. 3, p. 375, 2004. doi: 10.1016/j.tvj1.2004.10.013.

MILLS, D.S.; NANKERVIS, K. Comportamento equino. São Paulo. Roca, 2005. 213p.

NICOL, C.J. Equine estereotypies. In: HOUPT, K.A. (Ed). Recent advances in companion animal behaviour problems. Ithaca, 2000. Disponível em: <http://www.ivis.org/advances/Behavior Houpt/nicol/chapter_frm.asp?LA=1>. Acesso em: 02 nov. 2009.

VIEIRA, A.R.A. Prevalência e desgaste anormal dos dentes incisivos e incidência de cólica em eqüinos estabulados apresentando distúrbios de comportamento: análise de um total de 407 equinos. 2006. 47f. Dissertação (Mestrado em Ciência Animal) - Escola de Veterinária, Universidade Federal de Viçosa, MG. 